\title{
Memory and forgetting processes with the firing neuron model
}

\author{
D. Świetlik¹, J. Białowąs², A. Kusiak³ , D. Cichońska \\ ${ }^{1}$ Intrafaculty College of Medical Informatics and Biostatistics, Medical University of Gdansk, Poland \\ ${ }^{2}$ Department of Anatomy and Neurobiology, Medical University of Gdansk, Poland \\ ${ }^{3}$ Department of Periodontology and Oral Mucosa Diseases, Medical University of Gdansk, Poland
}

[Received 17 February 2018; Accepted: 23 April 2018]

The aim of this paper is to present a novel algorithm for learning and forgetting within a very simplified, biologically derived model of the neuron, called firing cell (FC). FC includes the properties: (a) delay and decay of postsynaptic potentials, (b) modification of internal weights due to propagation of postsynaptic potentials through the dendrite, (c) modification of properties of the analog weight memory for each input due to a pattern of long-term synaptic potentiation. The FC model could be used in one of the three forms: excitatory, inhibitory, or receptory (ganglion cell). The computer simulations showed that FC precisely performs the time integration and coincidence detection for incoming spike trains on all inputs. Any modification of the initial values (internal parameters) or inputs patterns caused the following changes of the interspike intervals time series on the output, even for the $10 \mathrm{~s}$ or $20 \mathrm{~s}$ real time course simulations. It is the basic evidence that the FC model has chaotic dynamical properties. The second goal is the presentation of various nonlinear methods for analysis of a biological time series. (Folia Morphol 2018; 77, 2: 221-233)

Key words: spiking neuron model, learning, long-term synaptic potentiation, forgetting, nonlinear time series analysis

\section{INTRODUCTION}

Learning, memory and forgetting are the essential properties of even the most primitive nervous system or brain. In humans many organic or psychiatric diseases strongly impair these functions (i.e. Alzheimer disease, schizophrenia, epilepsy, even stress) $[16,17$, $19,23,34,50]$. And we have as yet no satisfactory explanations for the basic mechanisms of these mental disorders. In many cases, additional knowledge could also emerge from computational models of single neurons or particular brain networks.

In neural networks, on a high level of biological realism, computation within the timing of individual spikes (action potentials) must be used. After the arrival of an action potential at the excitatory synapse we have an embarrassment of riches in which synaptic plasticity (inputs weights changes is the technical term) does occur. The biological neurons such as pyramidal cells in the hippocampus or cortex show various types of long-term synaptic potentiation (LTP) or depression (LTD) with presumably the dendritic location of their mechanisms throughout the N-methyl-D-aspartate (NMDA) glutamate receptors $[5,6,35,44]$. Currently available detailed simulators (Neuron, Genesis) of Hodgkin-Huxley neurons allow the maintenance of circuits consisting of thousands or even millions of cells, but in the case of simulations of large neural networks, the complexity of the neurons mathematical description with differential equations requires computational power which is hardly available $[9,25,54]$. For example Hendrickson

Address for correspondence: Dr. D. Świetlik, Intrafaculty College of Medical Informatics and Biostatistics, Medical University of Gdansk, ul. Dębinki 1, 80-211 Gdańsk, Poland, tel: +48 5834814 90, e-mail: dariusz.swietlik@gumed.edu.pl 
et al. [24] use, for the simulation of more than one million cells of the dentate gyrus of the rat brain, a computer cluster with a total of 4,040 processor cores and a maximum theoretical performance of 38.82 teraflops.

Simplified models of the integrate-and-fire (IF) type, on the other hand, do not cover the main properties of dendritic processes [11, 21, 27]. The IF model provides a caricature of the capacitive nature of the cell membrane at the expense of a detailed model of the refractory process. The IF model satisfies a current balance equation together with the condition that whenever the neuron reaches a threshold, it fires and the membrane potential is immediately reset to some reset potential, Vreset.

The detailed models of plasticity processes are not suitable for building large scale networks, so the most used rule for such models is the spike time dependent plasticity (STDP) sometimes completed with details of intracellular calcium turnover $[8,21,42]$. The STDP algorithm is a pure phenomenological approach, it could be easily used within computer simulations or complicated electronic circuits but, the living nervous cells solve this problem in another way, mainly by LTP/ LTD mechanisms. Recently a tagging procedure for LTP/LTD mechanisms as well as a probabilistic spiking neuron model approach were described $[8,13,29]$.

We have proposed that in neural simulations the computationally-expensive model of ionic channels' conductances can be replaced with a phenomenological model based on a set of shift registers in such a way that no essential output properties are lost because of such a replacement $[3,4,22]$. Thus the communication between particular neurons in the modelled network could be purely digital within zero-one courses and uses the timing of the output spikes to transmit information with only restraint being refraction time. The model also avoids the necessity of solving of differential equations.

What should be the reason for any biologically plausible synaptic plasticity mechanism or algorithm? Surely, the increase of particular synaptic weights must be rapid enough for survival and the depotentiation significantly slower. The algorithm newly employed in the firing cell (FC) neuron model covers those conditions. Real cognition requires the ability to learn sequences of patterns, and the most recently learned pattern does not erase the previously learned ones. Thus the spiking neural networks should have mechanisms to avoid that catastrophic interference, but some degree of forgetting should be always active, in other cases the possibility of significant syn- aptic weights changes rapidly decreases. So, we will also present the influence of various forgetting quotes (forgetting coefficient [FQ]) on the complex dynamic behaviour of FC model after the initiation of LTP.

Despite of all the complex mechanisms in a living nervous cell, the working result is always an action potential fired at a precise time depending on the input spike trains pattern. And if any, even very simple, models show efficient properties of time integration, coincidence detection and synaptic plasticity for inputs patterns, it should be useful for future challenges in neural computation.

To move further in understanding of how an incredibly sophisticated conglomerate such as the brain per se functions dynamically, at least, it is imperative to know the dynamics of its' elements - neurons. Having an exhaustive knowledge of the behaviours of neurons should help us to deepen our understanding of the synergy of cooperative behaviours of populations of neurons, neuronal networks, in this bottomup approach [2, 39-41]. Dynamically, neurons are categorised and characterised by kinds and shapes of membrane potential oscillations. Waveforms of such oscillations have been proved to be useful for prediction and identification of various neurological deceases such as Parkinson's disease or epilepsy [55]. A neuron is often viewed as a dynamic system [26], which can be evaluated by a broad family of nonlinear methods to describe neuronal activity over time. But what parameters should be evaluated? In mature nervous cells, the intrinsic parameters and synapses are well established, thus only the input and output spike trains patterns (inter spike intervals [ISI] time series) and in some diseases the synapses themselves undergo changes. So, we will put forward the question of how the elevated and memorised weight of particular inputs can change the output spike train. Surprisingly we are not able to find any data for such simulations in the literature, so a comparison with the most popular models such as integrate and fire, leaky integrate and fire, or neuron simulator is difficult. In this paper we will present a detailed formal and mathematical description of the FC model and some new methods of nonlinear analysis employed for the evaluation of simulations results.

\section{MATERIALS AND METHODS}

Mathematical presentation of the firing cell

For this presentation of the FC neuron model we adopted the general schema of hippocampal py- 
ramidal cell. Most of excitatory synapses are located among the long apical dendrite; the inhibitory synapses form mainly a "basket" around the cell body. The dendrite is modelled within a course of compartments and each has an excitatory or inhibitory synapse. The programme clock step of the presented model equals $0.5 \mathrm{~ms}$ of the simulated real time. First we create tables of shift registers; they form an array of looking up buffer tables. For excitatory inputs (glutamate receptors): AMPA - E (k,i), NMDA-M $(k, i)$, and for inhibitory inputs (GABA receptors): I(k,i) whereas " $k$ " is the number of the dendritic compartment, " $\mathrm{i}$ " is a number of an area in a particular register table. Each area simulates $0.5 \mathrm{~ms}$ of real time and initially is filled with the value of resting potential ( $R e P=-80 \mathrm{mV})$. The first position contains the actual value of postsynaptic potential calculated using (2.1), (2.2) and (2.3) for further computations within functions from (2.5) and (2.8).

Synaptic function - SF(t) calculates, after the arrival of an action potential on a particular synapse, the typical time courses of excitatory or inhibitory postsynaptic potentials (EPSP or IPSP) and furthermore adjusted in terms of parameters calculated from functions: Adaptation (2.2) and Memory (2.6) to the appropriate register table. It could be in simply linear form (2.1), but other forms as double exponential alpha function are also possible for use.

$$
S F(t)= \begin{cases}0 & t=t_{s d} \\ \frac{A_{M A X}}{t_{r}}\left(t-t_{s d}\right) & t_{s d}<t \leq t_{r} \\ \frac{A_{M A X}}{t_{d}}\left[\left(t_{d}-\left(t-\left(t_{r}+t_{s d}\right)\right)\right)\right] & t_{r}<t \leq t_{d}\end{cases}
$$

Where: $t_{s d}-$ time of synaptic delay, $t_{r}-$ time of EPSP/IPSP rise, $t_{d}$ - time of EPSP/IPSP decay, 1 millisecond $(\mathrm{ms})=2$ steps of $\mathrm{i}$. Parameters for EPSP AMPA $_{\text {: }}$ : $A_{\text {MAX }}=5 \mathrm{mV}, \mathrm{t}_{\mathrm{sd}}=1 \mathrm{~ms}, \mathrm{t}_{\mathrm{r}}=2 \mathrm{~ms}, \mathrm{t}_{\mathrm{d}}=13 \mathrm{~ms}$, for EPSP $_{\text {NMDA: }}: A_{\text {MAX }}=1 \mathrm{mV}, t_{s d}=1 \mathrm{~ms}, t_{r}=2 \mathrm{~ms}, t_{d}=$ $13 \mathrm{~ms}$, and for IPSP ${ }_{\mathrm{GABA}}: \mathrm{A}_{\mathrm{MAX}}=-2.5 \mathrm{mV}, \mathrm{t}_{\mathrm{sd}}=1 \mathrm{~ms}$, $\mathrm{t}_{\mathrm{r}}=2 \mathrm{~ms}, \mathrm{t}_{\mathrm{d}}=10 \mathrm{~ms}$.

Adaptation - $A(k, i)$ (for excitatory inputs):

$$
\mathrm{A}(\mathrm{k}, \mathrm{i})=\frac{(\text { ReP-Rsp })-(\operatorname{ReP}-\mathrm{S}(\mathrm{k}, \mathrm{i}))}{(\operatorname{ReP}-\mathrm{Rsp})}
$$

Where $S(i)$ is the actual value of summarised potential in the $\mathrm{k}$ compartment, ReP and Rsp parameters. $\mathrm{ReP}=-80 \mathrm{mV}$ (resting potential value), Rsp $=-10 \mathrm{mV}$ (reverse synaptic potential). It presents the possibility of reverse ionic flow through the membrane channels according to the Nernst equation.
Weight of compartment $\mathrm{k}-\mathrm{W}(\mathrm{k})$ (for excitatory inputs):

$$
\mathrm{W}(\mathrm{k})=\frac{1-\mathrm{LSW}}{\mathrm{NE}-1}(\mathrm{k}-\mathrm{NE})+1
$$

Where: LSW, NE - parameters; LSW (low significant weight) - weight of the most distal dendrite input. $\mathrm{NE}=13-$ a number of excitatory inputs. Thus within this function the cable properties of a modelled dendrite could be simply changed.

Function influence $-\operatorname{Inf}(a, b)$ of a compartment on b compartment (for excitatory inputs):

$$
\operatorname{Inf}(a, b)= \begin{cases}1-\frac{1-L S W}{(N-1)} & (a-b) b \geq a \\ 1-\frac{4}{N}(a-b) & (a-b) \leq \frac{N}{4} \\ 0 & \text { otherwise }\end{cases}
$$

$\mathrm{N}=$ total number of inputs and $a, b \in N$. This function allows for the calculation of summarised postsynaptic potential in a particular compartment (2.5) with respect to all others.

Summarised potential - $S(k ; i)$ in compartment $k$ (for excitatory inputs):

$$
\mathrm{S}(\mathrm{k}, \mathrm{i}+1)=\operatorname{ReP}+\sum_{\mathrm{m}=1}^{\mathrm{NE}}(\mathrm{E}(\mathrm{m}, \mathrm{i})-\mathrm{ReP}) \operatorname{Inf}(\mathrm{m}, \mathrm{k})
$$

Where: NE - number of excitatory inputs, $\operatorname{Inf}(m, k)$ influence of $m$ - compartment on $k, E(m ; 0)$ the actual value of the appropriate register.

Long-term synaptic potentiation (LTP), called, in short, Memory (Mem):

If $S(k, i)>$ CaMT then

$$
\operatorname{Mem}(\mathrm{k})=1+\ln \frac{\left(\mathrm{C}_{\mathrm{k}}(\mathrm{i})+1\right)}{6 \operatorname{clog}}
$$

CaMT $=-68 \mathrm{mV}$ (threshold for the removal of the $\mathrm{Mg}$ ion block for NMDA channels), $C(k$; i) time of memory for compartment $k$, clog parameter $=2.3026$. If we have simultaneously an action potential on excitatory input and opened NMDA channels due to enough depolarisation of the postsynaptic region (2.5), the LTP induction occurs; the weight of this synapse is being increased. Initiation of the cascade of biochemical reactions, those leading to LTP, depends of amount of calcium ions influenced throughout the opened NMDA channels.

We modelled that phenomenological event by power function:

$$
\text { power }=\text { powerA }(\mathrm{M}(\mathrm{k} ; \mathrm{i})-\mathrm{ReP})
$$

Whereas powerA $=9$ is a parameter, $M(k ; i)$ - actual value of SF(i) for EPSP(NMDA) in appropriate register. 
Time of memory duration $C(k ; i)$ :

$$
\mathrm{C}(\mathrm{k}, \mathrm{i}+1)=\mathrm{C}(\mathrm{k}, \mathrm{i})+\mathrm{e}^{\text {power }}-1
$$

Where: FQ; ReP — parameters. FQ — forgetting coefficient: it decreases, for the next simulation step, the calculated memory time of the programmed value. Clog, powerA, and FQ are numerical parameters, their changes allow confining the strength and time of LTP to biologically plausible values for any kind of modelled neuron.

\section{Art of work of firing cell model}

Input function $(\operatorname{InEx}(k))$ for excitatory and $\ln \operatorname{lnh}(k)$ for inhibitory synapses adds, after the arrival of an action potential (input $>0$ ), the values of the synaptic function (2.1) to the appropriate tables of shift registers $E(k ; i ; t), M(k ; i ; t)$ and $I(k ; i ; t)$, see below (2.9), (2.10) and (2.11). This sum is done with respect to the parameters calculated from functions (2.2) adaptation and (2.6) memory - LTP: thus the other functions are subsequently calculated and the threshold function is proofed. Simplified diagram see Figure 1.

$$
\begin{gathered}
\mathrm{E}(\mathrm{k} ; \mathrm{i} ; \mathrm{t}+1)=\mathrm{E}(\mathrm{k} ; \mathrm{i} ; \mathrm{t})+\mathrm{A}(\mathrm{k} ; \mathrm{i}) \operatorname{Mem}(\mathrm{k} ; \mathrm{i}) \mathrm{SF}(\mathrm{t}) \\
\mathrm{M}(\mathrm{k} ; \mathrm{i} ; \mathrm{t}+1)=\mathrm{M}(\mathrm{k} ; \mathrm{i} ; \mathrm{t})+\mathrm{SF}(\mathrm{t}) \\
\mathrm{I}(\mathrm{k} ; \mathrm{i} ; \mathrm{t}+1)=\mathrm{I}(\mathrm{k} ; \mathrm{i} ; \mathrm{t})+\mathrm{SF}(\mathrm{t})
\end{gathered}
$$

Summarised postsynaptic potential in neuron (PSP):

$$
\operatorname{PSP}(i+1)=\operatorname{ReP}+\sum_{\mathrm{m}=1}^{\mathrm{NE}} \mathrm{W}(\mathrm{k})\left(\mathrm{E}_{\mathrm{m}, 0}(\mathrm{i})-\mathrm{ReP}\right)+\sum_{\mathrm{m}=1}^{\mathrm{NE}}\left(\mathrm{I}_{\mathrm{m}, 0}(\mathrm{i})-\mathrm{ReP}\right)
$$

Where: $W(k)$ - weight of compartment $k, E(k ; i), I(k ; i)$ are the actual values of the tables from register $E$ and $I$ calculated with (2.9), (2.11), whereas the values of Adaptation (2.2) and Memory (2.6) are from a previous programme step. NE and $\mathrm{NI}$ are numbers of excitatory and inhibitory inputs. Threshold function for action potential: threshold $=-50 \mathrm{mV}$ :

$$
\text { out }=\left\{\frac{\text { oPSP }<\text { threshold }}{1 \text { PSP } \geq \text { threshold }}\right.
$$

Refraction is modelled for the time up to $2 \mathrm{~ms}$ by a reset of PSP value (2.11) for inhibitory input registers $\mathrm{I}(\mathrm{k} ; \mathrm{i})$ to the resting potential and shifting the voltage in the register tables $E(k ; i)$ for excitatory inputs according to the:

$$
\operatorname{ADRV}(\mathrm{k})=\operatorname{ReP}-\frac{(\mathrm{ReP}-\mathrm{RePB})(\mathrm{NE}-\mathrm{k})}{\mathrm{NE}}
$$

ADRV - AntiDromic Reset Voltage function (2.14); RePB is a parameter - voltage to which the most distal dendrite compartment is resetting $(-79 \mathrm{mV})$, $\mathrm{ReP}=-80 \mathrm{mV}$; NE - number of excitatory inputs.

Shifting of registers $E ; \mathrm{M}$; I; occurs at the end of each simulation step and the values in all registers tables are shifted as follow (th-threshold):

$\forall_{\mathrm{keN}} \forall_{\mathrm{ic}(0,31)} \mathrm{E}_{\mathrm{k}, \mathrm{i}}(\mathrm{t}+1)=\left\{\begin{array}{l}\mathrm{E}_{\mathrm{k}, \mathrm{i}+1}(\mathrm{t}), \text { for PSP }<\text { Threshold } \\ \text { ReP, for PSP } \geq \text { Threshold }\end{array}\right.$

$\forall_{\mathrm{keN}} \forall_{\mathrm{i}(0,3)} \mathrm{M}_{\mathrm{k}, \mathrm{i}}(\mathrm{t}+1)=\left\{\begin{array}{l}\mathrm{M}_{\mathrm{k}, \mathrm{i}+1}(\mathrm{t}), \text { for PSP }<\text { Threshold } \\ \text { ReP, for PSP } \geq \text { Threshold }\end{array}\right.$

$\forall_{k e N} \forall_{i e(0,31)} I_{k, i}(t+1)=\left\{\begin{array}{l}I_{k, i+1}(t), \text { for PSP }<\text { Threshold } \\ I_{k, i}(t), \text { for PSP } \geq \text { Threshold }\end{array}\right.$

\section{Summary of parameters used for equations (2.1)} to (2.17)

We define the numbers of Excitatory and Inhibitory inputs with their precise locations on the dendrite, then the values and time courses of postsynaptic potentials, threshold and refraction time values, and two basic physiological values - resting potential and synaptic reversal potential as commonly accepted in any book of physiology. AMAX parameters for SF(t) (2.1) were defined precisely for proper work of model with 16 inputs. We can change it to model various large of synapses as in biology. The numerical parameters for phenomenological modelled functions (2.4), (2.6), (2.7), (2.8) and (2.14) were determined with initial trials for optimal model behaviour.

\section{Other programmes used and download}

For statistical evaluation of output action potentials time series (ISI series) the StatSoft, Inc. STATISTICA (data analysis software system), version 13.1 (licensed for Medical University of Gdansk), MatLab The MathWorks, Inc. (licensed for Technical University of Gdansk), free VRA (visual recurrence analysis) programme of Kononow and free source code from Kreutz et al. [31] for ISI-distance measurement were used.

(http://web.archive.org/web/20070131023353/http:/ /www.myjavaserver.com/nonlinear/vra/download.html) (http://wwwold.fi.isc.cnr.it/users/thomas.kreuz/ sourcecode.html).

The demo version of the FC simulator can be downloaded from the site: (http://medinf.gumed. edu.pl/383.html). Using the demo version one can 


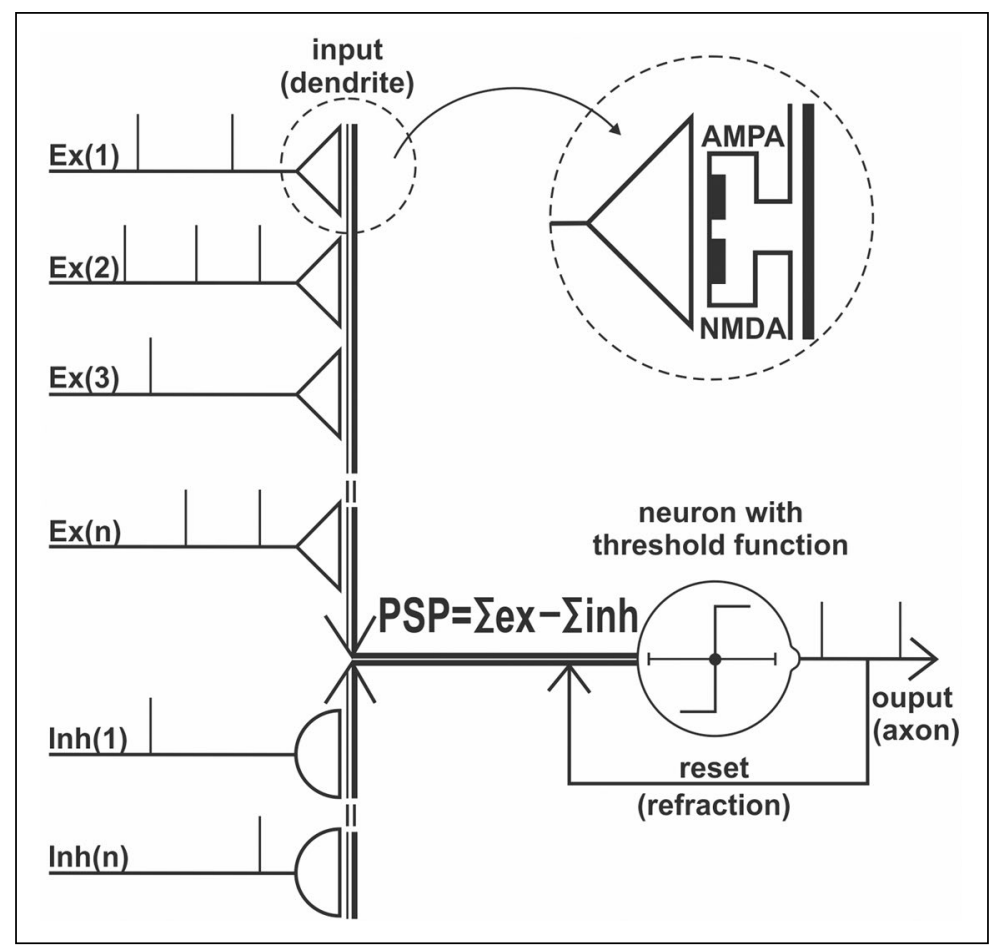

Figure 1. Simplified diagram of firing cell (FC)-Neuron Model. In the dotted circle the so called spine coincidence detector for weight changes based upon the biological mechanism of the long term synaptic potentiation (LTP). AMPA and NMDA — kinds of biological receptors; Ex excitatory; Inh — inhibitory inputs; PSP — summarised postsynaptic potential, calculated as the difference between sums of all excitatory (Eex) and inhibitory (Einh) inputs.

select the type of visualisation, open two tables comprising the values of initial parameters and inputs frequencies; the values could can be then accepted or changed before starting the simulation. For the free full version download under academic license contact the authors. The programme runs under Windows $X P$, 7,8 and 10 operating systems.

\section{RESULTS}

We configured the FC in such a way that it had 13 excitatory and 3 inhibitory inputs, each independently programmed. Biologically plausible frequencies for all inputs spike trains were arbitrary chosen as in the previously related papers $[3,4,22]$. For LTP induction the excitatory inputs 7,8 and 9 were stimulated at $100 \mathrm{~Hz}$ for $400 \mathrm{~ms}$ from the 2nd second of simulation. Such stimulation is closely similar to the LTP-inducing protocols from the work of Bliss and Lomo [7]. Simulations presented by Figures 2, 3 and 4 were performed with the $F Q=1$. The simulations from Figure 5 present the influence of increased FQ on output spike-trains. In all tested cases the frequency of action-potential generation and the values of LTP increased after the training procedure. The LTP values increase also within the neighbouring synapses in relation to those which were stimulated, so the FC model shows clearly the heterosynaptic LTP (Fig. 2C).

We have also tested the above configuration of $\mathrm{FC}$ without any LTP inducing protocol and we have found the increased frequency of output and some LTP induction from the 3rd second of simulation (Fig. 2D). This is due to the fact that at the end of the $2 \mathrm{nd}$ second there was a very narrow coincidence of some neigh-boring excitatory inputs. And the first noted increase of the LTP values occurs without that the cell fires any action potential. Within the used input frequencies we have periodically narrow coincidence of impulses on neighbouring excitatory synapses; it is the supporting cause for slowly but steady increase of LTP.

For the demonstration of regular, periodic or chaotic behaviour of a spiking neuron, as well as for the multiple neural spike train data analysis, various methods can be used. We present the analysis of the data utilising 2-dimensional phase space graphs with the time evolution of trajectory and with the aid of VRA, developed by Eugene Kononov, which was selected from various methods of recurrence plot analysis [36]. 


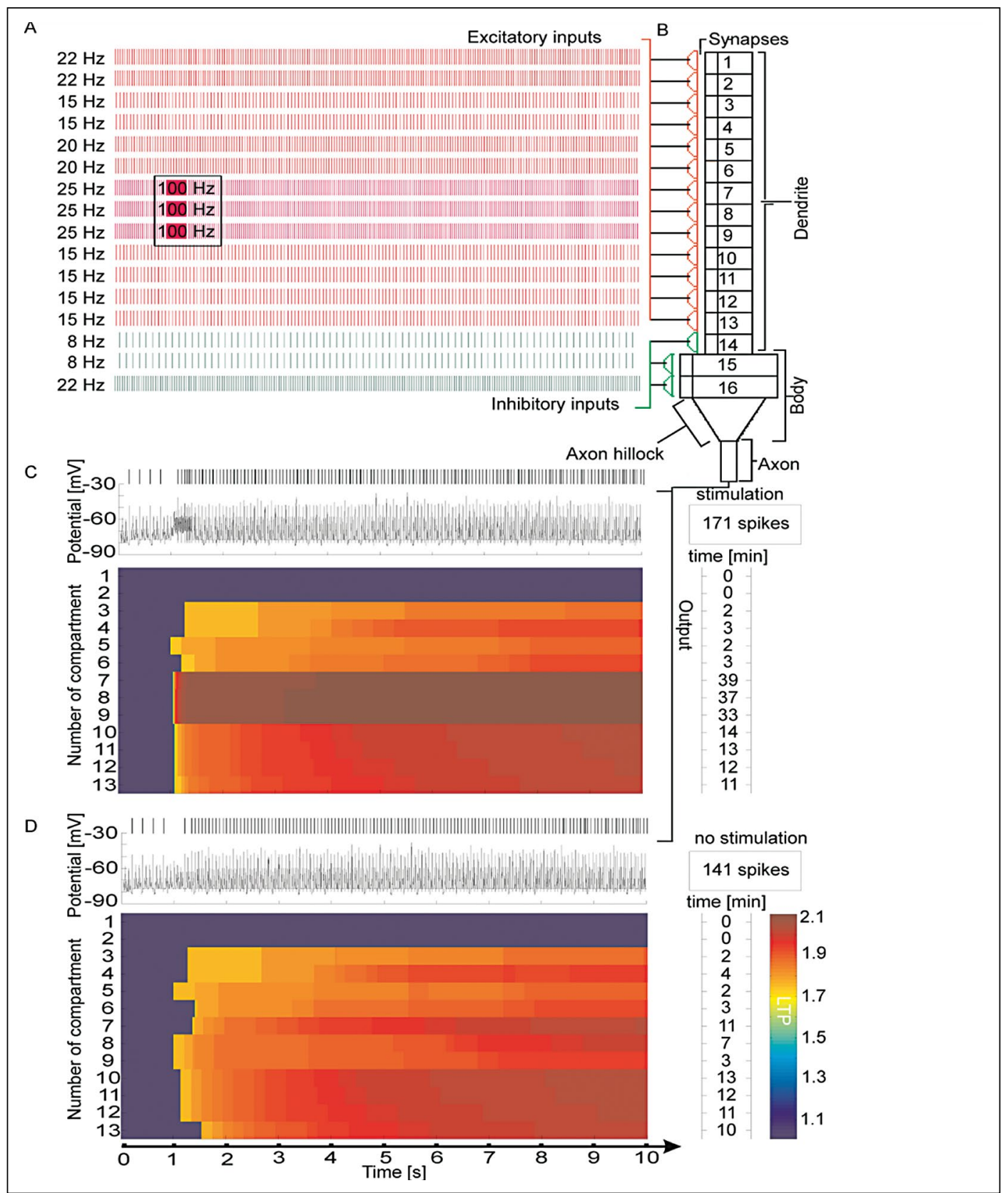

Figure 2. Basic $10 \mathrm{~s}$ real time simulation of firing cell $(\mathrm{FC})$ with low significant weight $(\mathrm{LSW})=0.2$, forgetting coefficient $(\mathrm{FO})=1$. A. All input and output spike trains. On the left particular input frequencies, the position of $100 \mathrm{~Hz}$ stimulation illustrated by red rectangles. Red excitatory, green inhibitory inputs; B. FC model configuration. A small rectangle below synapse means postsynaptic thickenings; C. Changes in long term synaptic potentiation (LTP) values shown at $200 \mathrm{~ms}$ intervals for all excitatory synapses during the simulation with LTP inducing protocol with output spikes train and time course of summarised post-synaptic potential at the top. D. Values of summarised post-synaptic potential, output spike train and LTP during the simulation without LTP inducing protocol. Time [min]-forecast time of duration LTP at the end of simulation.

The experiment without LTP inducing protocol Figure 2D shows, that after completing $2 \mathrm{~s}$ of simulation the increased output frequency stabilises at the value $15.039 \pm 0.104 \mathrm{~Hz}$ near to the most significant input frequency $15.033 \mathrm{~Hz}$ programmed for 6 excitatory inputs $(15 \mathrm{~Hz}$ adopted for $0.5 \mathrm{~ms}$ programme steps). The trajectory in phase-space converges up to
$8 \mathrm{~s}$ to a nearly point attractor. From the left panels on Figure 3A, $C$ it can be concluded that the LTP induction facilitates the additional firing in concordance with less significant inputs frequencies. This is clearly visible throughout on-line observation of the simulation (see video: medinf.gumed.edu.pl/383.html). Thus the FC changes output spike pattern during simulation 


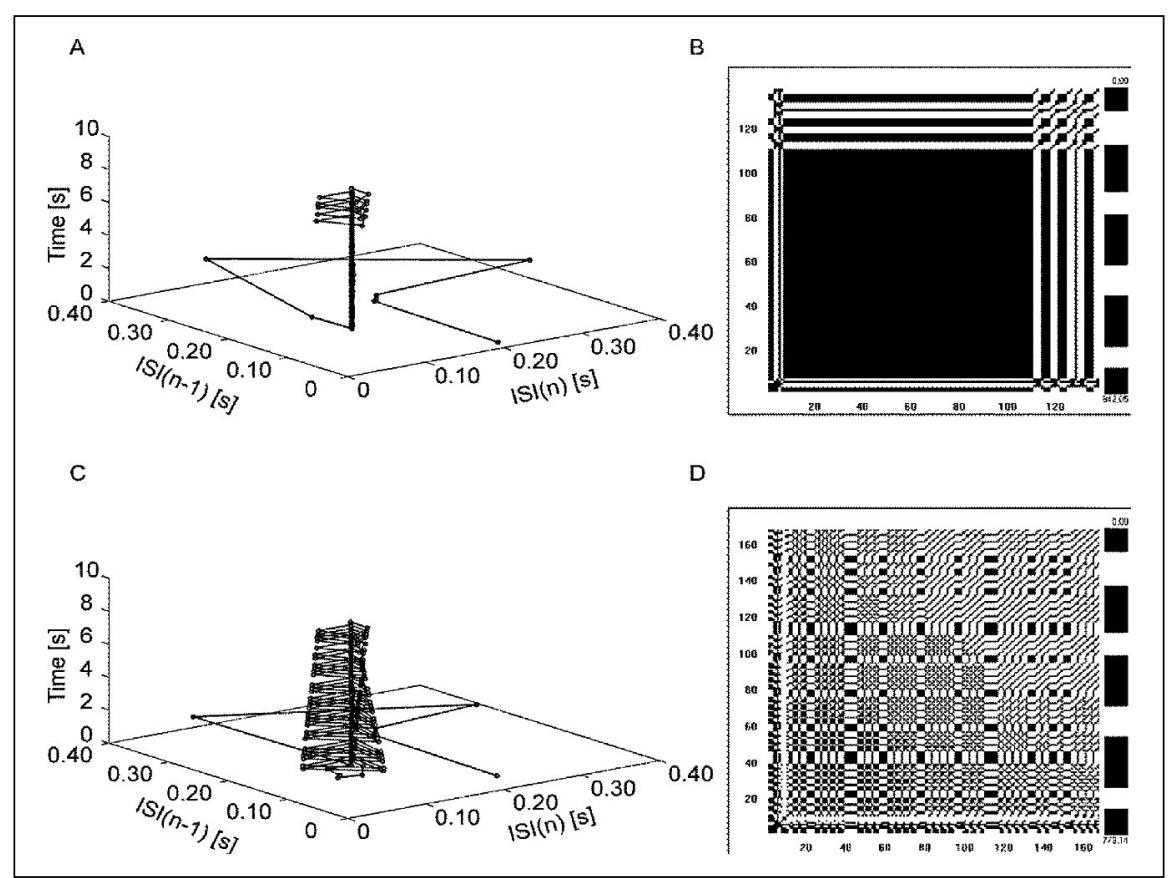

Figure 3. The nonlinear analysis of simulations from Figure 2; without long term synaptic potentiation (LTP) inducing protocol (A, B) and with ones (C, D). Left panels: 2-dimmensional phase space graph of inter-spike intervals; ISI(n), ISI(n-1), with time evolution of trajectory according to perpendicular axis z. Time units in seconds [s]. Right panels: recurrence plots with visual recurrence analysis (VRA), which show all Euclidean distances between data points from the 3-dimmensional phase space with time delay 1 (grey-tone version of original VRA basic colour scale). The coloured pixels are symmetrically distributed to diagonal.

from nearly regular to quasi-periodical with unstable orbits in the phase space.

To evaluate the capability of FC model, coincidence discrimination the set of simulations was performed, in which the inputs series of spikes on single excitatory inputs (input 10: $15 \mathrm{~Hz}$ and input 5: $20 \mathrm{~Hz}$ ) were shifted with delay $0.5 \mathrm{~ms}$. The same computations were done within FC model; both with and without the LTP inducing protocol. We present the influence of LTP increase on output spike trains with a method of ISI-distance measurement discovered by Kreuz et al. [31] in Figure 4A, B. The measure becomes zero when there are no differences and approaches 1 if the firing rates as compare both trains, achieve infinitely high differences. For a difference of $0.5 \mathrm{~ms}$ on single spike ISI-distance equals 0.000202 . The obtained values of ISI-distances Figure 4B indicate a moderately high pattern of differences between compared spike trains. The experiments show a greater differentiation and higher values thus better properties for coincidence detection in simulation with LTP induction protocol. It becomes clear from the values of the diagonals in Figure 4B, which compare corresponding simulations from both series. The results resemble some data from physiological experiments, in which the induction of LTP significantly increased coincidence detection for synapses on distal dendrites [56].

The results of the experiments with various FQ values confirmed the biological plausibility of FC model as an information-processing-related mechanism. In all tested series the frequency of action-potential generation and the values of LTP increased after the training with LTP inducing protocol. On the left panels of Figure 5 we show time evolution of trajectory in 2-dimensional phase space of ISI measurements for simulations with increased FQ values, as selected from an extensive series for FQ values 1 up to 100. As we compare trajectories from Figure 5 with those from Figure 3, for forgetting quotes above 10, trajectory tends to the same attractors courses as in the basic simulation without induction of LTP, which indicate a closely regular behaviour pattern. With lower forgetting quotes we observed basic regular behaviour, which irregularly switches to quasi-periodical with greater frequency. And with increased FQ values such changes occur rarely. Thus the FC model could be considered as very complicated iterative system with a tendency toward various types of chaotic behaviour. 


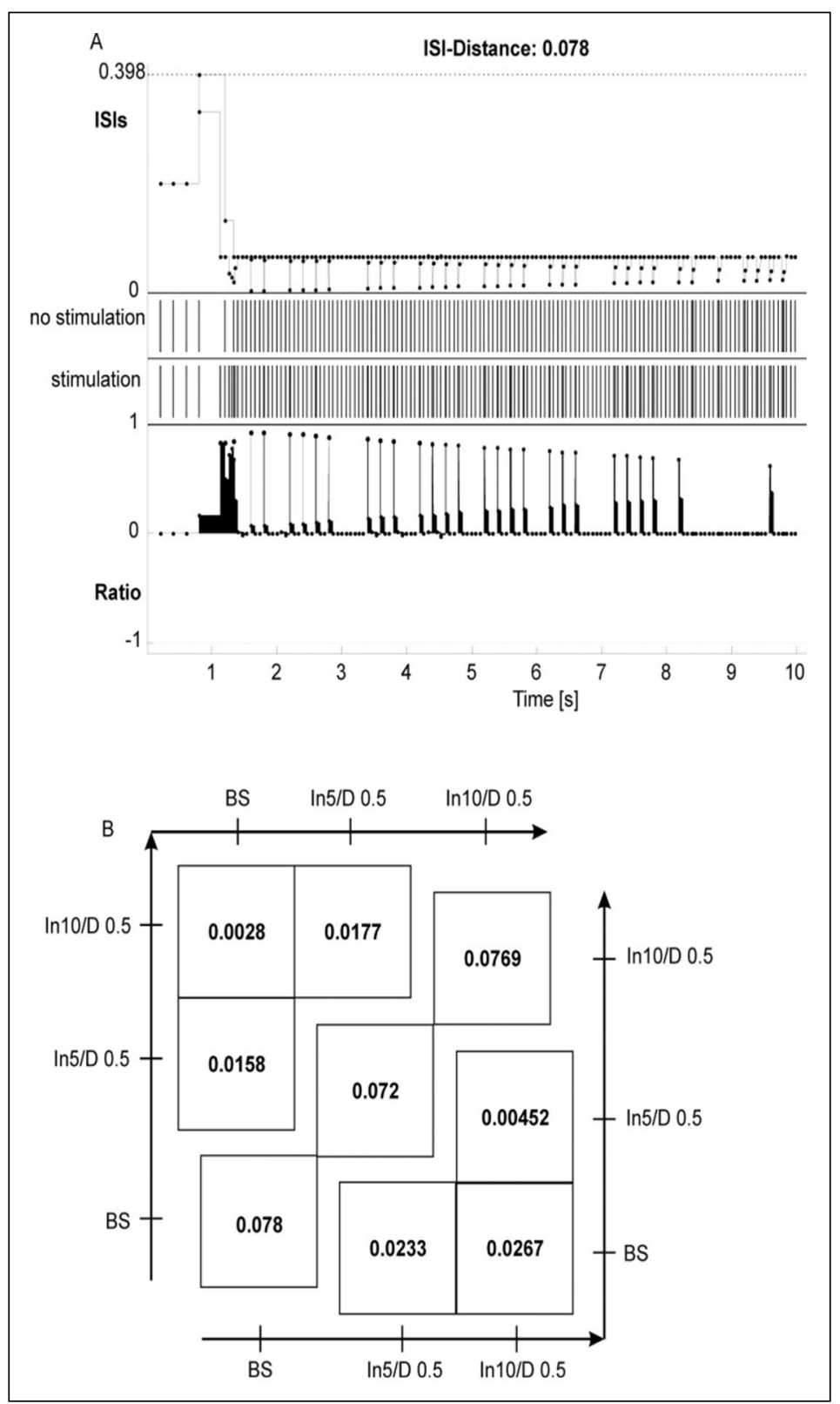

Figure 4. Influence of inputs frequency delays on coincidence detection, forgetting coefficient $(F 0)=1$. A. Inter-spike intervals (ISI) distance comparison of firing cell (FC) behaviour without comparisons for experiments with and without induction protocol for long term synaptic potentiation (LTP). In both experiments the delay of the spike series of $0.5 \mathrm{~ms}$ consecutively on input 5 and 10 synapses was tested. On the diagonal values of ISI-distances respectively for both induced LTP and with inducing stimulation. The distance value of 0.078 suggests a moderate difference. B. Matrix of possible ISI-distance series of simulations, below diagonal values for series with LTP induction, above those from series without $100 \mathrm{~Hz}$ stimulation (BS — basic simulations without input delays, In5-D 0.5 and In10-D 0.5 — particular inputs with delay $0.5 \mathrm{~ms}$ ).

Right panels from Figures 3 and 5 are recurrence plots with VRA, which show all Euclidean distances between data points from the 3-dimensional phase space graphs with colour scale (Basic or Froth).

\section{DISCUSSION}

The biologically plausible modelling of synaptic plasticity is a very complex and difficult process. The change of the synaptic weight could be triggered by single $1 \mathrm{~ms}$ action potential, but the consecutive long-term potentiation lasts many hours or days and within repeated supporting retrieval even months or years. For practical reasons the simulation algorithms for network and learning rules should be as simple as possible for minimal acceptable biological plausibility. The STDP algorithm for synaptic weights changes 


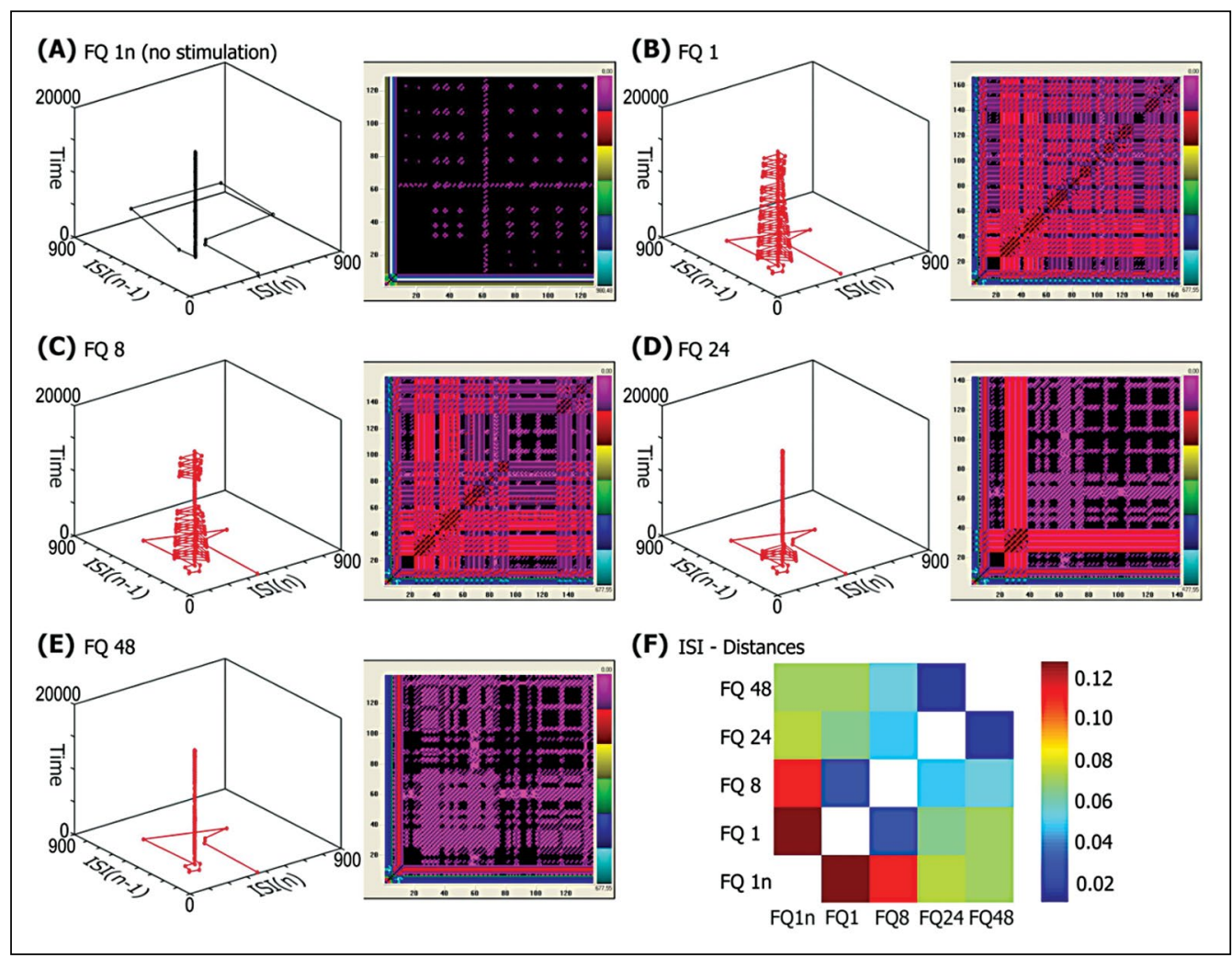

Figure 5. Another set of simulations. Phase space trajectory graphs for inter-spike intervals (ISI) measurements (with low significant weight $[L S W]=0.2$ ). Time axes with units at $0.5 \mathrm{~ms} ; 20,000$ means $10 \mathrm{~s}$ of simulation. A. For basic simulation without long term synaptic potentiation (LTP) inducing protocol and forgetting coefficient (FO) = 1. B, C, D, E. Simulations with the FO 1, 8, 24, 48, subsequently. The training of $400 \mathrm{~ms}$ at $100 \mathrm{~Hz}$ was with action potential spike trains on the 7th, 8th and 9th input from $2 \mathrm{~s}$. On the right visual recurrence analysis (VRA) plots for each simulation in the Froth special colour scale. The increased forgetting diminishes the elevated LTP learning effect. F. Matrix of ISI-distance measurements for 5 presented simulations from panels $A, B, C, D, E$.

$[14,42]$ could be regarded as far evolution of the old Hebb rule requires precise timing of presynaptic versus postsynaptic spikes. But recently there have been concerns about accuracy of such an algorithm $[10,18]$.

If an action potential reaches the synapse and the subsynaptic region is depolarised enough, does the calcium ions influx begin immediately and causes LTP. According to STDP rule the neuron should wait for the next postsynaptic spike to decide whether or not to turn on the LTP or LTD. Is it biologically plausible for higher inputs frequencies with tens of synapses?

Thus the presented LTP related algorithm solves such problems; it works on the dendrite level, independently for each compartment in concordance with canonical form of the sc. Spine coincidence detector [52], and in precise concomitance with the history of all inputs patterns. Because we have not any structural mechanism for the decrease of synaptic weights in FC, the simple FQ was introduced for biologically plausible working of the model; so the induced LTP has a finite time of duration.

Other research $[3,4]$ showed that in the short finite time (15 ms-time of EPSP course) the number of ISI combinations at the same synapse with regard to refraction is relatively small and the same for the modelled biological neuron, as in our FC model. Refraction relates to how after firing an action potential the neuron cannot immediately fire another one. The ability for further firing must be restored throughout ionic flows at the axon hillock and this requires time - the refraction time.

Briefly, if an action potential arrives at a synapse, the consecutive change of postsynaptic potential remains disposable to our model or neuron for further computation for a time of duration of typical EPSP or IPSP. For EPSP it is within FC $15 \mathrm{~ms}$ (30 clock steps). The number of ISI combinations depends on the number of action potentials, with regard to refraction, that arrived at the same synapse during this time. If we 
assume the difference of one clock step at one interval as significant for further computing, it can be calculated 6272 combinations from one spike to densely burst of 8 spikes. If we assume $1 \mathrm{~ms}$ as a significant difference, then we have 623 combinations. For 16 inputs - synapses of FC the total number of variations should be evaluated from the equation:

$$
\mathrm{V}^{\mathrm{k}}{ }_{\mathrm{n}}=\mathrm{n}^{\mathrm{k}}
$$

where $\mathrm{n}$ is the above calculated number of combination within the single output-input line equals 6272 and $k$ the number of synapses [3]. The calculated variation equals 627216 . And what variation could be calculated for 12,000 synapses on the hippocampal pyramidal cell or for 200,000 synapses on Purkinje cerebellar cell? Such calculations show all the most significant constrains for any further computational modelling. Before writing the presented work we have performed about 500 simulations each with another combination of inputs frequencies and initial model settings, but even for such simplified model as FC, we have for $10 \mathrm{~s}$ of simulation a total googol of variations. Coworkers of Mohemmed [37] have been presented SPAN - a spiking neuron that is able to learn associations of arbitrary spike trains in a supervised fashion allowing the processing of spatiotemporal information encoded in the precise timing of spikes. It allows for recognition of presented over 200 synapses spike patterns during $200 \mathrm{~ms}$ simulation. But it is a supervised learning rule and several hundreds of simulations do not cover all the variations possible for 200 to 600 hundreds of synapses even with that short time.

In each computer model the possibility of submillisecond coincidence detection should be as precise as the smallest modelled time step due to use of real values for computation. In biological neurons various membrane currents need some time to elevate potential, so the precision could be smaller, but the mathematical models of the dendrite show also the ability to perform sub-millisecond coincidence detection [53]. Therefore the high rate of coincidence detection should be a significant advantage of FC. We hope with further experimentation to find the set of initial values which should be most suitable for very accurate discrimination of various inputs patterns. The existence of chaos processes in brain networks is well confirmed and thus they should also exist in accurate models of neural tissue [30, 46]. Chaos processes could not be precisely predicted and for multiple inputs data and for longer time it is practically impossible to find any analytical solution. The Izhikevich model, as well as many others similar ones like Hindmarch-Rose (HRM), various Leaky Integrate and Fire (LIF), or even Spike Response Models (SRM) consists of simple differential equations with only a few parameters. It seems easy to define a set of parameters, and then feed a week additional input signal in order to investigate the "routes to chaos" or "stochastic resonance phenomena" $[2,40,41]$. When a model of even minimal biological plausibility is being built, it must present with any efficient learning mechanism (algorithm). As for the mature living neuron cells a defined set of internal parameters (in the model referred to as initial values) is stable, although there could be some differences for various brain areas. Thus, we have defined the numbers of Excitatory and Inhibitory inputs together with their precise locations on the dendrite, then the values and time courses of postsynaptic potentials, threshold and refraction time values, and two basic physiological values - resting potential and synaptic reversal potential. Using these above parameters we can introduce for model any algorithm of weight change (learning) for the inputs (synapses). In order to initiate the simulation we must define a signal for all excitatory inputs. This is " $I$ " in the Ishikevich equations.

With 16 inputs altogether the FC model shows at last tens of thousands possible variations of the inputs pattern. We have only selected a very few of those with the biologically plausible frequencies. Thereafter we could evaluate the output spikes trains. How the output spikes train can change after the weight elevation at particular input (learning, LTP)? We performed more than a thousand simulations with the single neuron, as well as with a small network of ten neurons connected as in the CA1 hippocampal area of the mammalians brain. Any changes in the initial values (internal parameters) or inputs patterns caused the following modifications of the ISI time series on the output, even for the $10 \mathrm{~s}$ or $20 \mathrm{~s}$ real time course simulations. It is the basic evidence, that the $\mathrm{FC}$ model has a chaotic dynamical properties.

There some remarks for the stochastic resonance phenomenon from the biological standpoint. It was first discovered in 1950 by Bernhard Langenbeck [33]. As of today such diagnostic method is widely used, The physical white noise signal enhances the ability of inner ear receptors to respond.

It is a well-known psychological phenomenon that certain emotions such as curiosity or fear enhance the 
ability to learn and memorise new patterns [47]. This is not a simple equivalent of accessory weak noise input, the pyramidal neurons in the cerebral cortex or the hippocampus become additional inputs from the excited emotion areas such as amygdaloid body [15]. It could be interpreted as an indirect supervised learning algorithm. Nevertheless in analysed simulations of FC all 16 inputs are programmed, so the "background noise" was still presented. The basic mechanism for Long Term Potentiation induction to synapses is the calcium ions influx throughout NMDA channels $[5,6$, $35,44]$. Such an ionic influx can occur only after removal of magnesium ions blockade of the NMDA channel by enough depolarisation of postsynaptic region. An instant depolarisation of the subsynaptic region depends on the history of inputs patterns, and so any accessory input in any form or shape could potentially enhance the ability to learn. It could be considered as a remote equivalent of the stochastic resonance phenomenon. As a cardinal mechanism involved in the learning process within the nervous tissue, the above should be employed in every complex or simplified neuronal model.

The presented nonlinear methods of ISI analysis are quite universal and could be used with any binarised time series of data, i.e. for evaluation of changes in heart rhythm and prognosis [1]. The FC model allows modifications of parameters from various functions and the changing of the cable properties of the dendrite with LSW values and so use them for modelling various types of excitatory or also inhibitory neurons. It is also possible to modify input functions so that they collaborate with the receptors in a way as ribbon synapse and use the FC for the modelling of ganglion cells [51].

It is the possibility of extracting the pure information processing algorithm from any other biological background (channels, membranes). We avoid using any of Hodgkin-Huxley, Integrate and Fire or Spike Timing Dependent Plasticity formalisms. Thus the basic concept of our model was derived directly from the theory of transistors with floating gates and capacitor coupling $[48,49]$ as well as from computer language and the simply models of all biological details considered within hitherto models of neuron. So the equations (2.1) to (2.17) are shown as in the source code, with parameters confined to biologically plausible values. The result is a mathematically consistent circuit that matches main prominent features of the living nervous cell, i.e. time integration, coincidence detection, learning with inputs weights changes and forgetting processes. Such circuits could be used to model nonlinear dynamic systems, as an alternative to the traditional differential equations approach. As shown within Figure 2, we are able to repeat by computer the canonical experiment of Bliss and Lomo from 1973 [7] for induction of long term synaptic potentiation in the rabbit brain hippocampus.

Such simplified methods are not adequate for the modelling of detailed neurophysiologic data concerning various receptors and channels, with even hundreds of synapses, but should have many advantages for long time examinations of various recognition or decision processes. George and Hawkins [20] use, instead of neurons, the HTM nodes abstracted as coincidence detectors and a mixture of Markov chains, and demonstrate the application of the derived circuits for the modelling of the subjective contour effect and pattern recognition. Thus the developed FC-circuit can be used for modelling physiological phenomena and for deriving testable predictions about the brain.

Especially, the circuit model within shift registers is well suited for spatiotemporal computing, which has recently seen more interest [12]. Such simple mathematical of biologically inspired processors that work like living neural circuits, And they could be more easily implemented in hardware such as Neuron-MOS Transistor of Shibata and Ohmi $[48,49]$ and projecting its' use for neuron circuits built with nanodot technology described by Morie et al. [38]. The memristive hardware emulation of synaptic plasticity is still a point of great interest $[28,32,45]$.

\section{CONCLUSIONS}

Computational abstractions could be used to model the cognitive activity taking place in the brain. The problem would be then somehow collect these processes, and embody them into biological neural networks. Thus the hardware implementation of FCcircuit could be a step forward to solve the above question with regard to the dominant significance of synchrony between various brain regions in consciousness phenomenon [43]. As already mentioned for the model of 1 million interconnected neurons a cluster of over 4000 processors should be used [24]. It seems that one can only hope for the future to bring along new technology enabling more efficient analog processors to be harnessed for computational research. 


\section{REFERENCES}

1. Babinec $P$, Kučera $M$, Babincová $M$. Global characterization of time series using fractal dimension of corresponding recurrence plots: from dynamical systems to heart physiology. Harmon Fractal Image Anal. 2005; 1: 87-93.

2. Barrio R, Martínez MA, Serrano $S$, et al. Macro- and micro-chaotic structures in the Hindmarsh-Rose model of bursting neurons. Chaos. 2014; 24(2): 023128, doi: 10.1063/1.4882171, indexed in Pubmed: 24985442.

3. Bialowas J, Grzyb B, Poszumski P. Firing Cell: An Artificial Neuron with Long-Term Synaptic Potentiation Capacity. 2005 International Conference on Neural Networks and Brain. 2005; 3, doi: 10.1109/icnnb.2005.1614874.

4. Bialowas J, Grzyb B, Poszumski P. Firing cell: an artificial neuron with a simulation of long-term-potentiationrelated memory. ISAROB. 2005; 11: 731-734.

5. Bliss TVP, Collingridge GL, Morris RGM. Synaptic plasticity in health and disease: introduction and overview. Philos Trans R Soc Lond B Biol Sci. 2014; 369(1633): 20130129, doi: 10.1098/rstb.2013.0129, indexed in Pubmed: 24298133.

6. Bliss TV, Collingridge GL. A synaptic model of memory: long-term potentiation in the hippocampus. Nature. 1993; 361 (6407): 31-39, doi:10.1038/361031a0, indexed in Pubmed: 8421494.

7. Bliss T, Lømo T. Long-lasting potentiation of synaptic transmission in the dentate area of the anaesthetized rabbit following stimulation of the perforant path. J Physiol. 1973; 232(2): 331-356, doi: 10.1113/jphysiol.1973.sp010273, indexed in Pubmed: 4727084.

8. Borges RR, Borges FS, Lameu EL, et al. Effects of the spike timing-dependent plasticity on the synchronisation in a random Hodgkin-Huxley neuronal network. Commun Nonlinear Sci Numer Simul. 2016; 34: 12-22, doi: 10.1016/j.cnsns.2015.10.005.

9. Bower JM, Beeman D. The Book of Genesis - Exploring Realistic Neural Models with the GEneral NEural SImulation System. Genesis. 2003; 2003, doi:10.1016/\$13646613(02)01915-0, indexed in Pubmed: 300835.

10. Brea J, Gerstner W. Does computational neuroscience need new synaptic learning paradigms? Curr Opin Behav Sci. 2016; 11: 61-66, doi:10.1016/j.cobeha.2016.05.012.

11. Brette R, Rudolph M, Carnevale T, et al. Simulation of networks of spiking neurons: a review of tools and strategies. J Comput Neurosci. 2007; 23(3): 349-398, doi: 10.1007/ s10827-007-0038-6, indexed in Pubmed: 17629781.

12. Buonomano DV, Maass W. State-dependent computations: spatiotemporal processing in cortical networks. Nat Rev Neurosci. 2009; 10(2): 113-125, doi: 10.1038/nrn2558, indexed in Pubmed: 19145235.

13. Clopath C, Büsing L, Vasilaki E, et al. Connectivity reflects coding: a model of voltage-based STDP with homeostasis. Nat Neurosci. 2010; 13(3): 344-352, doi: 10.1038/ nn.2479, indexed in Pubmed: 20098420.

14. Clopath C, Ziegler L, Vasilaki E, et al. Tag-trigger-consolidation: a model of early and late long-term-potentiation and depression. PLoS Comput Biol. 2008; 4(12): e1000248, doi: 10.1371/ journal.pcbi.1000248, indexed in Pubmed: 19112486.

15. Diano M, Tamietto M, Celeghin A, et al. Dynamic changes in amygdala psychophysiological connectivity reveal dis- tinct neural networks for facial expressions of basic emotions. Sci Rep. 2017; 7: 45260, doi: 10.1038/srep45260, indexed in Pubmed: 28345642.

16. Duch W. Therapeutic implications of computer models of brain activity for Alzheimer disease. JMIT. 2000; 5: 27-34.

17. Duch W. Computational models of dementia and neurological problems. Methods Mol Biol. 2007; 401: 305-336, doi: 10.1007/978-1-59745-520-6_17, indexed in Pubmed: 18368373.

18. Feldman DE. The spike-timing dependence of plasticity. Neuron. 2012; 75(4): 556-571, doi: 10.1016/j.neuron.2012.08.001, indexed in Pubmed:22920249.

19. Finkel L. Neuroengineering Models of Brain Disease. Annu Rev Biomed Eng. 2000; 2(1): 577-606, doi: 10.1146/annurev.bioeng.2.1.577, indexed in Pubmed: 11701524.

20. George D, Hawkins J. Towards a mathematical theory of cortical micro-circuits. PLoS Comput Biol. 2009; 5(10): e1000532, doi:10.1371/journal.pcbi.1000532, indexed in Pubmed: 19816557.

21. Gerstner W, Kistler WM. Spiking neuron models: single neurons, populations. Plasticity. Book. 2002; 494, doi: 10.2277/0511075065, indexed in Pubmed: 12443231.

22. Grzyb B, Bialowas J. Modeling of LTP-related phenomena using an artificial firing cell. Lecture Notes in Computer Science. 2006; 4232: 90-96.

23. Hasselmo ME, McClelland JL. Neural models of memory. Curr Opin Neurobiol. 1999; 9(2): 184-188, indexed in Pubmed: 10322183.

24. Hendrickson P, Yu G, Song D, et al. Interactions between inhibitory interneurons and excitatory associational circuitry in determining spatio-temporal dynamics of hippocampal dentate granule cells: a large-scale computational study. Front Syst Neurosci. 2015; 9, doi: 10.3389/ fnsys.2015.00155, indexed in Pubmed: 26635545.

25. Hines M. A program for simulation of nerve equations with branching geometries. Int J Biomed Comput. 1989; 24(1): 55-68, doi: 10.1016/0020-7101(89)90007-x, indexed in Pubmed: 2714879.

26. Hodgkin AL, Huxley AFA. Quantitative description of membrane current and its application to conduction and excitation in nerves. J Physiol. 1952; 117: 500-554, doi: 10.1016/ S0092-8240(05)80004-7, indexed in Pubmed: 12991237.

27. Izhikevich EM. Which model to use for cortical spiking neurons? IEEE Trans Neural Netw. 2004; 15(5): 1063-1070, doi: 10.1109/TNN.2004.832719, indexed in Pubmed: 15484883.

28. Kang DH, Jun HG, Ryoo KC, et al. Emulation of spike-timing dependent plasticity in nano-scale phase change memory. Neurocomputing. 2015; 155: 153-158, doi: 10.1016/j. neucom.2014.12.036.

29. Kasabov N. To spike or not to spike: a probabilistic spiking neuron model. Neural Netw. 2010; 23(1): 16-19, doi: 10.1016/j. neunet.2009.08.010, indexed in Pubmed: 19783402.

30. Korn H, Faure P. Is there chaos in the brain? II. Experimental evidence and related models. Comptes Rendus Biol. 2003; 326(9): 787-840, doi:10.1016/j.crvi.2003.09.011, indexed in Pubmed: 14694754.

31. Kreuz T, Haas JS, Morelli $A$, et al. Measuring spike train synchrony. J Neurosci Methods. 2007; 165(1): 151-161, doi: 10.1016/j.jneumeth.2007.05.031, indexed in Pubmed: 17628690 . 
32. La Barbera S, Vuillaume D, Alibart F. Filamentary switching: synaptic plasticity through device volatility. ACS Nano. 2015; 9(1): 941-949, doi:10.1021/nn506735m, indexed in Pubmed: 25581249.

33. Langenbeck B. Geräuschaudiometrische Diagnostik. Die Absolutauswertung: Archiv für Ohren-, Nasen- und Kehlkopf-Heilkunde. 1950; 158: 458-471.

34. Llorens-Martin M, Blazquez-Llorca L, Benavides-Piccione R, et al. Selective alterations of neurons and circuits related to early memory loss in Alzheimer's disease. Front Neuroanat. 2014; 8, doi: 10.3389/fnana.2014.00038, indexed in Pubmed: 24904307.

35. Malenka RC, Bear MF. LTP and LTD: an embarrassment of riches. Neuron. 2004; 44(1): 5-21, doi: 10.1016/j.neuron.2004.09.012, indexed in Pubmed:15450156.

36. Marwan N, Carmen Romano M, Thiel M, et al. Recurrence plots for the analysis of complex systems. Physics Reports. 2007; 438(5-6): 237-329, doi:10.1016/j.physrep.2006.11.001, indexed in Pubmed: 18291439.

37. Mohemmed A, Schliebs S, Matsuda S, et al. Span: spike pattern association neuron for learning spatio-temporal spike patterns. Int J Neural Syst. 2012; 22(4): 1250012, doi: 10.1142/S0129065712500128, indexed in Pubmed: 22830962

38. Morie $T$, Matsuura $T$, Nagata $M$, et al. A multinanodot floating-gate MOSFET circuit for spiking neuron models. IEEE Transactions On Nanotechnology. 2003; 2(3): 158-164, doi: 10.1109/tnano.2003.817221.

39. Nagornov R, Osipov G, Komarov M, et al. Mixed-mode synchronization between two inhibitory neurons with postinhibitory rebound. Commun Nonlinear Sci Numer Simul. 2016; 36: 175-191, doi: 10.1016/j.cnsns.2015.11.024.

40. Nobukawa S, Nishimura H, Yamanishi T. Chaotic Resonance in Typical Routes to Chaos in the Izhikevich Neuron Model. Sci Rep. 2017; 7(1): 1331, doi:10.1038/s41598-01701511-y, indexed in Pubmed: 28465524.

41. Nobukawa S, Nishimura H, Yamanishi T, et al. Analysis of Chaotic Resonance in Izhikevich Neuron Model. PLoS One. 2015; 10(9): e0138919, doi:10.1371/journal. pone.0138919, indexed in Pubmed: 26422140.

42. Nowotny T, Rabinovich MI, Abarbanel HDI. Spatial representation of temporal information through spiketiming-dependent plasticity. Phys Rev E Stat Nonlin Soft Matter Phys. 2003; 68(1 Pt 1): 011908, doi: 10.1103/ PhysRevE.68.011908, indexed in Pubmed: 12935177.

43. Pereira A, Ferreira Almada L. Conceptual spaces and consciousness: integrating cognitive and affective processes. Int J Mach Conscious. 2011; 03(01): 127-143, doi: 10.1142/s1793843011000649.

44. Raymond CR. LTP forms 1, 2 and 3: different mechanisms for the "long" in long-term potentiation. Trends Neurosci.
2007; 30(4): 167-175, doi:10.1016/j.tins.2007.01.007, indexed in Pubmed: 17292975.

45. Saïghi S, Mayr C, Serrano-Gotarredona T, et al. Plasticity in memristive devices for spiking neural networks. Front Neurosci. 2015; 9, doi:10.3389/fnins.2015.00051, indexed in Pubmed: 25784849.

46. Segundo J. Nonlinear dynamics of point process systems and data. Int J Bifurc Chaos. 2003; 13(08): 2035-2116, doi: $10.1142 / \mathrm{s} 0218127403007886$.

47. Shen L, Wang M, Shen R. Affective e-Learning: Using "emotional" data to improve learning in pervasive learning environment related work and the pervasive e-learning platform. Educ Technol Soc. 2009; 12: 176-189.

48. Shibata T, Ohmi T. An intelligent MOS transistor featuring gate-level weighted sum and threshold operations. International Electron Devices Meeting [Technical Digest]. IEDM. 1991 : 919-922, doi: 10.1109/iedm.1991.235276.

49. Shibata T, Ohmi T. Neuron MOS binary-logic integrated circuits. I. Design fundamentals and soft-hardware-logic circuit implementation. IEEE Transactions on Electron Devices. 1993; 40(3): 570-576, doi: 10.1109/16.199362.

50. Siekmeier PJ, Hasselmo ME, Howard MW, et al. Modeling of context-dependent retrieval in hippocampal region CA1: implications for cognitive function in schizophrenia. Schizophr Res. 2007; 89(1-3): 177-190, doi: 10.1016/j. schres.2006.08.007, indexed in Pubmed: 17055702.

51. Sikora MA, Gottesman J, Miller RF. A computational model of the ribbon synapse. J Neurosci Methods. 2005; 145(12): 47-61, doi:10.1016/j.jneumeth.2004.11.023, indexed in Pubmed: 15922025.

52. Sjöström PJ, Rancz EA, Roth A, et al. Dendritic excitability and synaptic plasticity. Physiol Rev. 2008; 88(2): 769-840, doi: 10.1152/physrev.00016.2007, indexed in Pubmed: 18391179.

53. Softky W. Sub-millisecond coincidence detection in active dendritic trees. Neuroscience. 1994; 58(1): 13-41, doi: 0.1016/0306-4522(94)90154-6, indexed in Pubmed: 8159289 .

54. Traub RD, Contreras D, Cunningham MO, et al. Singlecolumn thalamocortical network model exhibiting gamma oscillations, sleep spindles, and epileptogenic bursts. J Neurophysiol. 2005; 93(4): 2194-2232, doi: 10.1152/ jn.00983.2004, indexed in Pubmed: 15525801.

55. Uhlhaas PJ, Singer W. Neural synchrony in brain disorders: relevance for cognitive dysfunctions and pathophysiology. Neuron. 2006; 52(1): 155-168, doi: 10.1016/j.neuron.2006.09.020, indexed in Pubmed: 17015233.

56. Xu NI. Coincidence detection of synaptic inputs is facilitated at the distal dendrites after long-term potentiation induction. J Neurosci. 2006; 26(11): 3002-3009, doi: 10.1523/ /jneurosci.5220-05.2006, indexed in Pubmed: 16540578. 